\title{
Design of Sliding Mode Speed Controller for Switched Reluctance Motor
}

\author{
Mohammed Moanes E. Ali and Yousif Khudhair Fakhir ${ }^{*}$ \\ Department of Electrical and Electronic Engineering, University of Technology, Baghdad, Iraq \\ Received 01 Jan 2018, Accepted 01 March 2018, Available online 04 March 2018, Vol.8, No.2 (March/April 2018)
}

\begin{abstract}
Switched Reluctance motors (SRM) can be classified into a group of multi-speed electrical motors. The low cost, rugged constructions and simple are feature advantages for this motor. The simplicity is the result of their torque generation principle, which known as variable reluctance principle. The SRM has many more features that have made it to be common for applications in commercial and industrial markets. The main disadvantage of SRM is the nonlinearity that it appears in their dynamics because of the magnetic saturation. It is required for SRM speed controllers to have features such as fast dynamic responses, parameter insensitivity and quick recovery from load disturbances. In this paper, a design of a robust sliding mode speed controller based on a nonlinear mathematical model is proposed. Matlab/Simulink software is used to simulate switched reluctance motor drive system under control of SMC. 6/8 switched reluctance motor has been taken as case study. The performance of proposed sliding mode control is tested at different load and speed conditions, and comparisons with conventional PI control for switched reluctance motor are presented. The sliding mode controller exhibits a better performance than the PI controller for all the studied cases.
\end{abstract}

Keyword: Switched Reluctance Motor; PI; Sliding Mode; Speed Control.

\section{Introduction}

The variable reluctance machine (VRM) has been proposed for multi-speed drive system in the 1960s (Sakurai, 2001). According to the development of power electronics and computer science, researches have been directed towards reluctance motors especially switched reluctance motors (SRM). SRM has the advantages of reliability and ability of four quadrants operation through a multi-speed range. The low cost, rugged constructions and simple are, also, feature advantage for this motor (TEJ Miller,1993) . These features have made SRM to be common for applications in commercial markets and industrial.

The SRM operates without any mechanical commutation components. It has doubly salient poles. Generally, the rotor is a simple construction made of magnetic iron, formed in such a way as to maximize the variation of inductance with position. Torque is generated by the tendency of its rotor to move to a position where the inductance of the active coil is maximized. A SRM has multiple teeth on the rotor poles and is capable of producing high torque. The motion may be linear or rotary, and the moveable part may be exterior or interior (Krishnan, 2001).

*Corresponding author's ORCID ID: 0000-0000-0000-0000 DOI: https://doi.org/10.14741/ijcet/v.8.2.3
The switched-reluctance motors suffer from high nonlinearity; this is due to the magnetic saturation. Therefore, it is necessary to operate this motor under control of nonlinear robust controller (Sakurai, 2001). Sliding mode control (SMC) is a type of control technique that can guarantee the stability of the nonlinear systems. The concept of sliding mode control uses a discontinuous control input in order to drag the state trajectory of the system to slide along a prespecified surface in the state space. This surface is named a sliding-manifold, represents the desired plant dynamics properties, such as tracking and stability to the origin. An advantage of sliding mode control is its robustness with respect to external disturbance and changes in system parameters (Yang Haiqing, et al, 1996).

In this paper, the mathematical model of the SRM is described and the sliding mode controller is proposed as a speed controller for this motor. The proposed design is simulated by using Mtlab/Simulink software, the SRM drive system is tested under various operating conditions and the results are compared with PI controller.

\section{Mathematical model of SRM}

Ignoring the mutual inductance between the phases, the equivalent circuit for the SRM can be developed as follows. 
The applied voltage to any phase is equal to:

$v=R_{S} i+\frac{d \lambda(\theta, i)}{d t}$

where $R_{S}$ is the resistance per phase, and $\lambda$ is the flux linkage per phase, the flux linkage given by:

$\lambda(\theta, i)=L(\theta, i) i$

where $\mathrm{L}$ is the inductance which is a function of phase current and rotor position. The phase voltage equation, then, is

$v=R i+L(\theta, i) \frac{d i}{d t}+i \frac{d \theta}{d t} \frac{d L(\theta, i)}{d \theta}$

$=R_{s} i+L(\theta, i) \frac{d i}{d t}+i \omega_{m} \frac{d L(\theta, i)}{d \theta}$

The induced emf, e, is obtained as:

$e=\frac{d L(\theta, i)}{d t} \omega_{m} i=K_{b} \omega_{m} i$

where $\mathrm{Kb}$ is an emf constant and it equals to:

$K_{b}=\frac{d L(\theta, i)}{d t}$

The emf constant is dependent on the operating point and it can be obtained at constant current at this point. From the voltage expression and the induced emf equation, the equivalent circuit of the SRM (only one phase) is shown in figure 1.

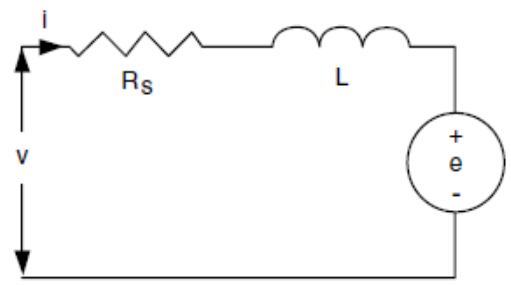

Figure 1: One-phase equivalent circuit of SRM

The power can be evaluated as:

$p_{i}=v i=R_{s} i^{2}+i^{2} \frac{d(\theta, i)}{d t}+L(\theta, i) i \frac{d i}{d t}$

Here, the last term is physically uninterruptable; to draw a meaningful inference, it may be form in terms of known variables as below:

$\frac{d}{d t}\left(\frac{1}{2} L(\theta, i) i^{2}\right)=L(\theta, i) i+\frac{1}{2} i^{2} \frac{d L(\theta, i)}{d t}$

and power equation is:

$p_{i}=R_{S} i^{2}+L(\theta, i) i+\frac{1}{2} i^{2} \frac{d L(\theta, i)}{d t}$

where $\mathrm{Pi}$ is the instantaneous input power. The input power is the sum of the resistive losses, the rate of change of the field energy, and the air gap power pa which is defined by $\left(L(\theta, i) \cdot i^{2} / 2\right)$. Substituting the time in terms of the rotor position and speed, with

$t=\frac{\theta}{\omega_{m}}$

in the air gap power results in:

$p_{a}=\frac{1}{2} i^{2} \frac{d L(\theta, i)}{d \theta} \frac{d \theta}{d t}$

The air gap power is given by:

$p_{a}=\omega_{m} T_{e}$

Electromagnetic torque is obtained by equations (11) and (12) as (Krishnan, 2001):

$T_{e}=\frac{1}{2} i^{2} \frac{d L(\theta, i)}{d \theta}$

\section{Design of SMC for SRM}

The design procedure of sliding mode control (SMC) can be described in the following two steps:

Step one: switching surface is formulated according to the error in the dynamic system.

Step two: control law is implemented in such way that it should force the system trajectories arrive to the sliding surface.

The main property of using sliding mode control is, once the system is in sliding phase, it doesn't effect to parameter variations and uncertain disturbances. The error in the dynamic system is related as:

$e(t)=\omega(t)-\omega_{m}(t)$

where $e(t)$ is the error of motor speed $\omega(t)$ with respect to reference speed $\omega_{m}(t)$. The sliding surface $\mathrm{S}$ is defined as:

$S=\dot{e}(t)+\lambda e(t) \quad \lambda>0$

$\lambda$ has to be positive and considerably large so that speed error e(t) will vanish exponentially and sufficiently fast on the hyper-plane $S=0$. Consider a Lyapunov function

$v(s)=\frac{s^{2}}{2}$

The basic idea in designing a sliding mode controller (SMC) is to use a control strategy for the plant input $\mathrm{u}(\mathrm{t})$ such that $\dot{V}(s) \leq 0$ regardless of the spatial position of the state $X(t)$. Then it can be asserted that the system state will be steer towards the sliding surface $\mathrm{s}$ $=0$ and reach it within finite time (Yang Haiqing, et al, 1996). 
The equations of SRM model are as follow:

$\frac{d i}{d t}=\left(\frac{\partial \lambda(\theta, i)}{\partial i}\right)^{-1}\left(u_{i}-R_{j} i_{j}-\omega \frac{\partial \lambda_{j}(\theta, i)}{\partial \theta} i_{j}\right)$

$\omega=\frac{d \theta}{d t}$

$\dot{\omega}=\frac{1}{J}\left(T_{e}-B \omega-T_{L}\right)$

By differentiating equation (19):

$\ddot{\omega}=\frac{1}{J}\left(\sum_{j=1}^{3} \frac{\partial T(\theta, i)}{\partial i} \frac{d i}{d t}+\omega \sum_{j=1}^{3} \frac{\partial T(\theta, i)}{\partial \theta}-B \dot{\omega}-\frac{d T_{L}}{d t}\right)$

$\ddot{\omega}=\frac{1}{J}\left(\sum_{j=1}^{3} \frac{\partial T(\theta, i)}{\partial i}\left(\frac{\partial \lambda(\theta, i)}{\partial i}\right)^{-1}\left(u_{i}-R_{j} i_{j}-\omega \frac{\partial \lambda_{j}(\theta, i)}{\partial \theta} i_{j}\right)+\right.$

$\left.\omega \sum_{j=1}^{3} \frac{\partial T(\theta, i)}{\partial \theta}-B \dot{\omega}-\frac{d T_{L}}{d t}\right)$

where $\frac{d T_{L}}{d t}$ the uncertain quantity. Now let's define $u(t)=V_{d c}$ [see Fig 2]. Obviously, $u(t)$ is the plant input and is to be controlled. The phase voltage $u_{i}$ can be written $\operatorname{as}_{i}=k_{j} u(t)$ where is ordered by the commutation signals.

$k_{j}=\left\{\begin{array}{c}1 \\ -1 \\ 0\end{array}\right.$

1 , when this phase must be turned on

-1 , when this phase must be turned off and

0 , when this phase must be turned off and $i_{j}=0$

Then (21) becomes

$\ddot{\omega}=\frac{1}{J}\left(\left[\sum_{j=1}^{3} \frac{\partial T(\theta, i)}{\partial \theta}-\sum_{j=1}^{3} \frac{\partial T(\theta, i)}{\partial i}\left(\frac{\partial \lambda(\theta, i)}{\partial i}\right)^{-1} \frac{\partial \lambda_{j}(\theta, i)}{\partial \theta} i_{j}\right] \omega-\right.$

$B \dot{\omega}+\sum_{j=1}^{3} \frac{\partial T(\theta, i)}{\partial i}\left(\frac{\partial \lambda(\theta, i)}{\partial i}\right)^{-1} k_{j} u+\dot{T}_{L}-$

$\left.\sum_{j=1}^{3} \frac{\partial T(\theta, i)}{\partial i}\left(\frac{\partial \lambda(\theta, i)}{\partial i}\right)^{-1} R_{j} i_{j}\right)$

$\ddot{\omega}=P_{1} \omega+P_{2} \dot{\omega}+P_{3} u+d(t)$

Where

$P_{1}=\frac{1}{J}\left[\sum_{j=1}^{3} \frac{\partial T(\theta, i)}{\partial \theta}-\sum_{j=1}^{3} \frac{\partial T(\theta, i)}{\partial i}\left(\frac{\partial \lambda(\theta, i)}{\partial i}\right)^{-1} \frac{\partial \lambda_{j}(\theta, i)}{\partial \theta} i_{j}\right]$

$P_{2}=-\frac{B}{J}$

$P_{3}=\frac{1}{J} \sum_{j=1}^{3} \frac{\partial T(\theta, i)}{\partial i}\left(\frac{\partial \lambda(\theta, i)}{\partial i}\right)^{-1} k_{j}$

$d(t)=\frac{1}{J}\left[\dot{T}_{L}-\sum_{j=1}^{3} \frac{\partial T(\theta, i)}{\partial i}\left(\frac{\partial \lambda(\theta, i)}{\partial i}\right)^{-1} R_{j} i_{j}\right]$

The derivative of Lyapunov function is:

$\dot{V}(s)=S \dot{S}=S[\ddot{e}(t)+\lambda \dot{e}(t)]=S\left[P_{1} \omega+P_{2} \dot{\omega}+P_{3} u+\right.$

$\left.d(t)-\dot{\omega}_{\text {ref }}+\lambda \dot{e}(t)\right]$

The control law

$(t)=-\frac{1}{P_{3}}\left[\left(\dot{\omega}_{r e f}+\lambda \dot{e}(t)\right)+P_{1} \omega+P_{2} \dot{\omega}+d(t)-\right.$

$\left.K_{s} \operatorname{sign}(S)\right]$
Where $K_{S}>0$ and the function $\operatorname{sgn}(\mathrm{S})$ is illustrated as:

$$
\operatorname{sgn}(S)=\begin{array}{r}
1 \text { if } s>0 \\
0 \text { if } s=0 \\
-1 \text { if } s<0
\end{array}
$$

Then equation (24) will become

$\dot{V}(S)=S\left[-\frac{K_{S}}{J} \operatorname{sgn}(S)\right]=-\frac{K_{S}}{J}|S| \leq 0$

From equation (27), $\dot{V}(s) \leq 0$ for all time t, but. This show that $V(S)$ is limited, so $\mathrm{S}$ is bounded. Now

$\int_{0}^{\infty}-\frac{K_{S}}{J} d t=V(S)-V(\infty) \leq V(0)$

Equation (28) indicates that $S$ is integral. Since $S$ is bounded, so $\lim _{t \rightarrow \infty} S=0$.

\section{The proposed Speed Controller of the SRM}

Based on the mathematical model of sliding mode speed controller, the proposed control system is structured as shown in figure 2. To evaluate the performance of proposed speed controller, Matlab/Simulink software environment is used. The Simulink model of SRM drive system is shown in figure 3 , and the details of the sliding mode controller are shown in Figure 4.

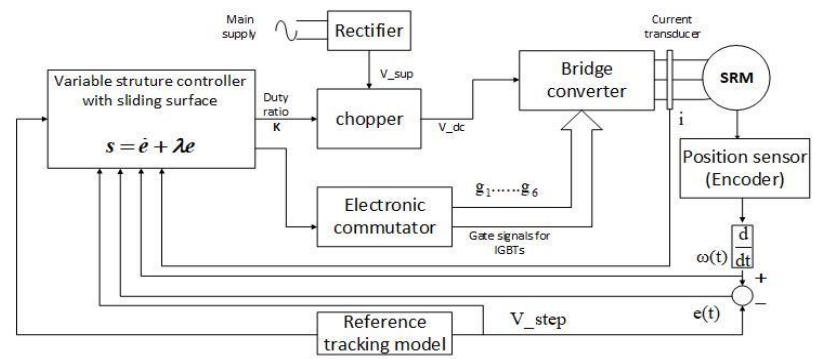

Figure 2 The structure of with sliding mode controller (SMC) for SR motor

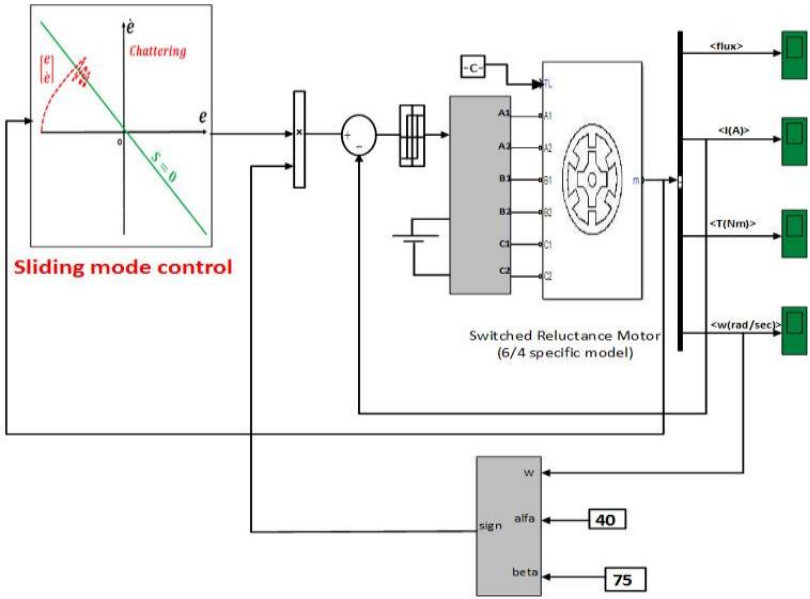

Figure 3 The simulation of the drive system based on SMC 


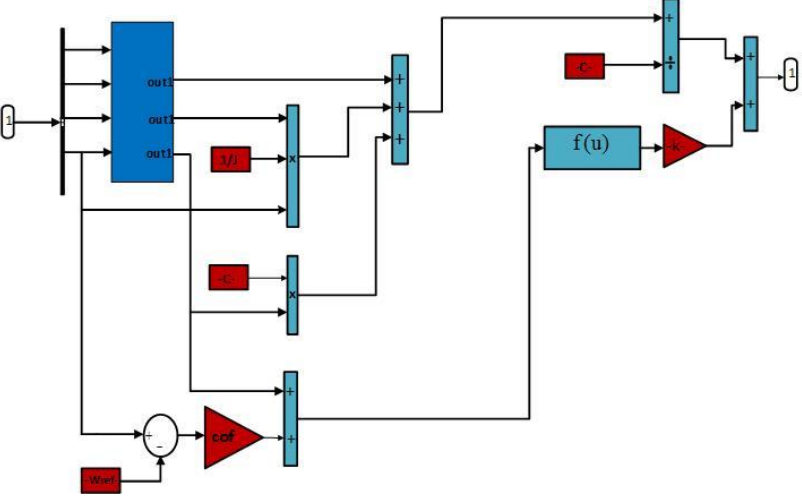

Figure 4 The details of the sliding mode speed controller

\section{The Results and Performance of the Sliding- Mode Controller}

The performance of the sliding mode speed controller is studied under various operating conditions and the results are compared with the traditional PI controller. The results of speed response tests at starting the SRM with different reference speed and load torque equal to zero (no-load) are shown in the next figures. The response speed of the motor at the reference speed of $100 \mathrm{red} / \mathrm{sec}$ is shown in figure 5 while the response at reference speed of $300 \mathrm{red} / \mathrm{sec}$ is shown in figure 6 . In figure 7 , the reference speed is changed from 300 to $600 \mathrm{rad} / \mathrm{sec}$ (rated speed) at time instant $=0.6 \mathrm{sec}$.

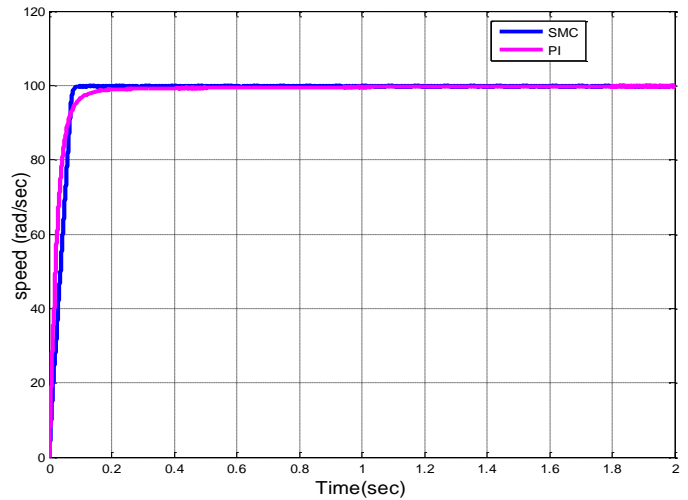

Figure 5 Speed response at reference speed 100 $\mathrm{rad} / \mathrm{sec}$ and no load

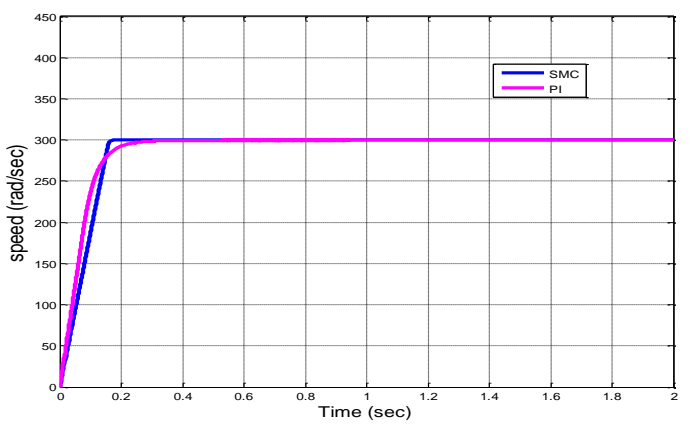

Figure 6 Speed response at reference speed 300 $\mathrm{rad} / \mathrm{sec}$ and no load

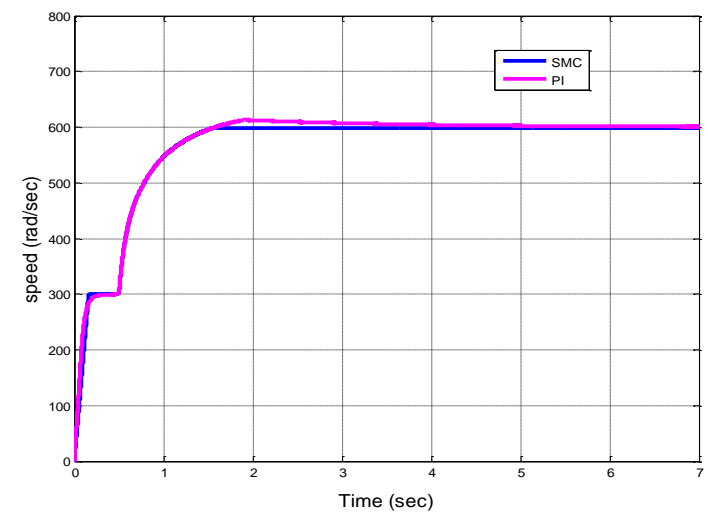

Figure 7 Speed response at changing the reference speed from 300 to $600 \mathrm{rad} / \mathrm{sec}$

From figure 5, figure 6 and figure 7, it is clear that the sliding mode controller shows excellent performance and it is extremely superior to the PI control. The sliding mode controller satisfies all the requirements, where there is no overshoot, the rise time is extremely fast and the steady-state error tends to be null.

Figure 8, figure 9 and figure 10 show the behavior of the motor under different load condition. In figure 8 the motor is subjected to constant load toque of 80 N.m. In figure 9 the torque is suddenly increase from 30 to $80 \mathrm{~N} . \mathrm{m}$ at time instant $0.8 \mathrm{sec}$, while in figure 10 the load torque is suddenly decrease from 80 to $0 \mathrm{~N} . \mathrm{m}$ at time instant $4 \mathrm{sec}$.

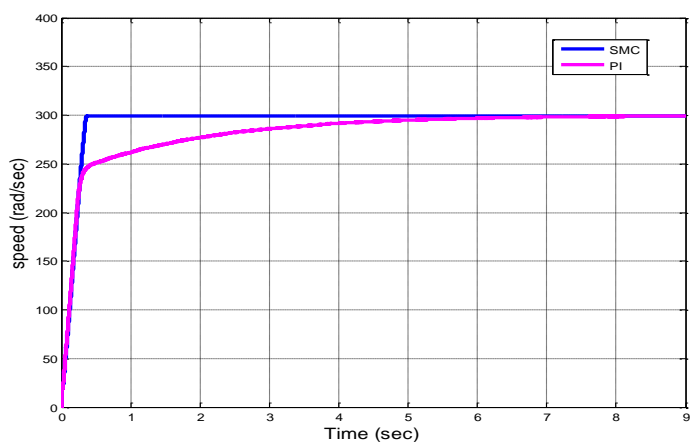

Figure 8 Speed response at load torque of 80 N.m

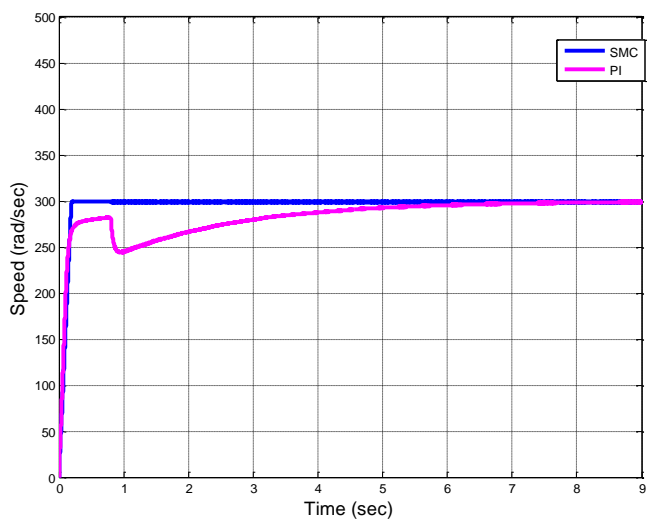

Figure 9 Speed response when the load is increase from 30 to 80 N.m 


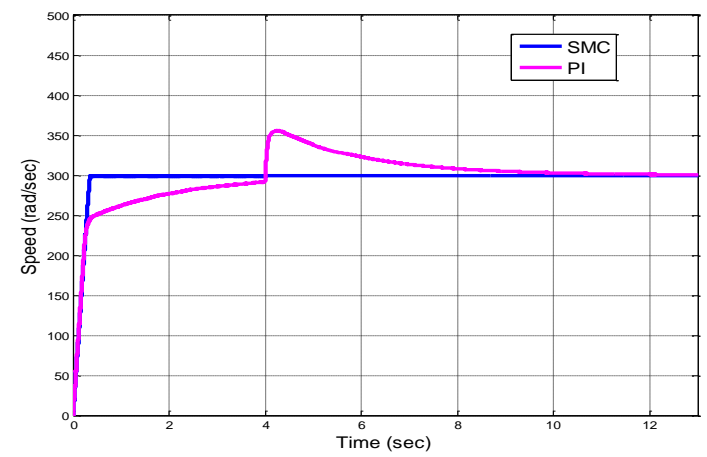

Figure 10 Speed response when the load is decrease from 80 to 0 N.m

From figure 8, figure 9 and figure 10, it can be concluded that SMC is more robust as compared to PI control, where the SMC has the ability to maintain speed without change even at significant changes in the load.

\section{Conclusions}

In this work, the sliding mode control is used as a speed controller for the switched reluctance motor; a comparison of performance is done between this controllers with the well-known PI controller, various operating conditions have been considered. The sliding mode controller exhibits a better performance than the PI controller for all the studied cases. The results of operating the sliding mode controller can be summarized as:
1) At starting the motor, the speed responses have no overshoot for all operating conditions.

2) The rising time is very short and it is shorter that of PI controller.

3) In a case of sudden change of reference speed, no overshoot appears.

4) The speed remains constant even there is a heavy change of load.

\section{Reference}

TEJ Miller (1993), Switched reluctance motors and their control. Magna Physics Pub and Claredon Press.Oxford.

Krishnan, Ramu (2001) Switched reluctance motor drives: modeling, simulation, analysis, design, and applications. CRC press

Khalil, Ahmed, and Iqbal Husain (2007) A fourier series generalized geometry-based analytical model of switched reluctance machines. IEEE transactions on industry applications 43.3: 673-684.

Kumar, S. Suresh, and J. Jayakumar (2016) Torque modeling of Switched Reluctance Motor using LSSVM-DE. Neurocomputing 211: 117-128.

$\mathrm{Xu}$, Zhan-Zhi, et al (2016) Sliding mode control of the planar switched reluctance motor for interference suppression. Industrial Electronics and Applications (ICIEA), IEEE 11th Conference on. IEEE, 2016

Wang, Shun-Yuan, Foun-Yuan Liu, and Jen-Hsiang Chou (2016) Design on Sliding Mode Controller with Adaptive Fuzzy Compensation for Switched Reluctance Motor Drive Systems. Computer, Consumer and Control (IS3C), International Symposium on. IEEE, 2016.

Atsuhiko Sakurai (2001) Sliding mode control of switched reluctance motors. National Library of Canada ,Bibliothéque nationaledu Canadadu. 